\title{
Modelo de decisiones compartidas en cáncer de mama. De la teoría a la práctica
}

\author{
Miriam de la Flor López \\ Universitat Rovira i Virgili \\ Miriamjose.delaflor@urv.cat
}

Maria Inmaculada de Molina-

Fernández

Universitat Rovira i Virgili.

Inmaculada.demolina@urv.cat

\author{
Alba Roca-Biosca \\ Universitat Rovira i Virgili \\ Alba.roca@urv.cat
}

\author{
Ramon Escuriet-Peiro \\ Servei Català de la Salut \\ rescuriet@gencat.cat
}

Juana Galera Ortega

Universitat Rovira i Virgili

jgalor@tinet.org

Lourdes Rubio-Rico

Universitat Rovira i Virgili

lourdes.rubio@urv.cat

Resumen: La decisión de reconstruirse o no la mama es personal y debe basarse en una buena información. En este artículo se presenta el proceso sistemático de desarrollo y resultado de una herramienta de ayuda a la toma de decisiones (HATD) digital para mujeres con cáncer de mama. Dicha herramienta plantea a la mujer las opciones disponibles ante una mastectomía, lo que constituye una novedad en el campo de las HATD al no disponerse en la literatura de ninguna berramienta de trabajo similar hasta el momento. Las politicas sanitarias deben promover la creación y utilización de instrumentos que favorezcan y potencien que los pacientes, con ayuda de los clinicos, se impliquen y tomen las decisiones sobre su salud de un modo informado.

Palabras clave: cáncer de mama; reconstrucción mamaria inmediata; reconstrucción mamaria diferida; herramientas de ayuda a toma de decisiones; decisiones compartidas; cuidados centrados en el paciente. 


\begin{abstract}
The decision to pursue breast reconstruction or not is a personal choice that should be based on solid information. In this article we present the systematic process of developing and building a digital shared decision-making tool for women with breast cancer. Before undergoing a mastectomy, women can use this tool to find out which options are available to them. It constitutes a novelty in the field of shared decision-making tools, as no similar work tool has been described in the literature to date. Health policies should promote the creation and use of instruments that favor and empower patients, with the clinician's help, to get involved and make informed decisions about their health.
\end{abstract}

Keywords: Breast cancer; immediate breast reconstruction; deferred breast reconstruction; decision-making supporting tools; shared decisions; patient-centered care. 


\section{El paciente en el centro del proceso asistencial. Liderazgo e impacto social}

En el ámbito de la salud, la calidad es un término claramente relacionado con la responsabilidad y el compromiso del profesional con el paciente, «lo mejor para él» (Mitchell, 2008). Este enfoque está relacionado con el principio de beneficencia y está asociado al correcto proceso de diagnóstico, tratamiento y cuidados. Por otro lado, el concepto de práctica clínica contempla muchos aspectos, como la información, las percepciones, los razonamientos y juicios, las decisiones de los profesionales, los procedimientos que usan y las intervenciones que aplican (Guyatt, 1991). El análisis de la práctica clínica muestra la existencia de gran variabilidad intra e interpersonal, es decir, la variabilidad en las decisiones de un mismo profesional o entre profesionales diferentes ante un mismo problema, de la cual se deriva la incertidumbre en la práctica clínica (Mira, 2006).

La variabilidad en la práctica clínica aumenta en las situaciones en las que existen varias posibilidades de diagnóstico o de tratamiento y ninguna se ha demostrado superior a las otras en resultados. Asimismo, la decisión en favor de cualquiera de las alternativas puede estar sujeta a diversos factores importantes según la escala de valores de cada paciente (Mendoza, 2006).

Las decisiones se deben tomar a partir de las preferencias y valores de cada persona. En determinadas situaciones o ante ciertos temas que se repiten en nuestras vidas, es probable que las preferencias y valores estén claros y, por tanto, las decisiones se pueden tomar de forma rápida o rutinaria. Cuando se trata de asuntos relativos a la salud, ya sea por la incertidumbre asociada o por tratarse de situaciones más infrecuentes, los valores y preferencias no suelen ser tan obvios y la elección exige sopesar detenidamente pros y contras. La deliberación previa a este tipo de decisión demanda un trabajo de introspección que requiere de ayuda estructurada por parte de un profesional (Abellán, 2008).

En las decisiones clínicas se debe ser muy cuidadoso, ya que se puede caer en la actuación médica exagerada o medical overuse, que no es más que una sobreutilización de recursos y procedimientos cuando estos pueden comportar efectos indeseables o ningún cambio en la situación clínica y que posiblemente los pacientes rechazarían si estuviesen bien informados (May, 2009).

En la literatura se define una buena decisión clínica (Ratliff, 1999) como aquella que se toma de forma compartida con un paciente correctamente informado, es decir, aquel que ha recibido la información que puede procesar sobre beneficios y riesgos de procedimientos y/o tratamientos, aclarando que no existe uno «mejor» per se, sino que la decisión depende de preferencias y valores personales. (Elwyn, 2000; O'Connor, 2002). 
En la atención a la salud existen diferentes modelos de relación que exigen distintos grados de interacción y adaptación entre profesionales y pacientes. En un extremo está el modelo paternalista o dirigido, en el cual la decisión la toma plenamente el profesional, que es quien dispone del saber y la tecnología necesarios para ello, e interpreta también las necesidades y deseos de los pacientes, que no participan en la decisión final (Emanuel, 1992). En el otro extremo está el modelo de decisiones informadas, en el cual el profesional pone a disposición del paciente toda la información relevante del caso, y deposita en él la responsabilidad de la decisión (Mendoza, 2006). En un punto intermedio se sitúa el modelo de decisiones compartidas. En él, el profesional proporciona información basada en la evidencia, el paciente expone sus dudas, preferencias y valores, y el profesional responde facilitando nueva información adaptada que permita encontrar la decisión que mejor se ajuste al caso, convirtiendo a los pacientes en participantes activos e informados (Charles, 1997). Se trata de un modelo de relación clínica respetuosa y deliberativa, que exige, además, conocimientos y habilidades de comunicación. Unos conocimientos y habilidades escasamente empleados hasta ahora, y que constituyen una auténtica novedad en el trabajo clínico diario (Couceiro, 2005). En resumen, una discusión detallada entre el paciente y el profesional, seguida de una recomendación centrada en el paciente, lleva a un proceso de decisiones compartidas de alta calidad (Mitchell, 2008). Sin ese paso, toda recomendación hecha es directiva y paternalista y ahoga el derecho de autonomía del paciente. Por otro lado, esa mirada hacia el paciente permite discriminar aquellos pacientes que se encuentran más cómodos con un estilo paternalista o directivo para respetarlo (Emanuel, 1992).

\section{La toma de decisiones compartida. El modo de empoderar al paciente}

La toma de decisiones compartida en salud es un tema que ha comenzado a generar interés en los últimos quince años. La definición más extendida lo presenta como un estilo de relación entre el profesional sanitario y el paciente, que se puede aplicar a todo tipo de interacción o de consulta, en que el profesional de la salud y el paciente comparten todas las etapas del proceso de toma de decisiones simultáneamente (Charles, 1997).

En la literatura encontramos muchos ejemplos de posibles estrategias para favorecer el proceso de decisiones compartidas en distintas instituciones y/o países, aunque, en esencia, todos incluyen los mismos aspectos a tener en cuenta (Stiggelbout, 2012). 
1. Desarrollar una relación de confianza con el paciente, ofreciendo empatía y calidez. Es importante que exista una comunicación fluida y facilitar el discurso del paciente.

2. Explorar y determinar las preferencias del paciente sobre la información que desea recibir (formato, cantidad...).

3. Explorar y determinar las preferencias del paciente acerca de su papel en la toma de decisiones (como el riesgo que quiere asumir o el nivel de participación tanto suya como de amigos o familiares). Algunos pacientes temen ser asertivos y que esto ponga en peligro la relación médico-paciente y derive en un cuidado de menor calidad. Por ello, los clínicos deberían invitar a los pacientes a participar, identificar qué es lo que estos necesitan para tomar las decisiones, apoyarles apropiadamente y ayudarles a tomar las decisiones cuando los pacientes prefieren delegar en ellos.

4. Responder a las ideas, preocupaciones y expectativas de los pacientes (como por ejemplo sobre las opciones de manejo de la enfermedad) atendiendo a las «señales» que estos proporcionan.

5. Identificar las opciones (incluidas las ideas y la información que el paciente pueda tener) y valorar la evidencia en el contexto del paciente individual; sin olvidar que no hacer nada también es una opción.

6. Presentar la evidencia y ayudar al paciente a reflexionar y a evaluar el impacto de las alternativas en relación con sus valores y preferencias.

7. Tomar o negociar la decisión en conjunto con el paciente y resolver posibles conflictos.

8. Acordar un plan de acción y preparar el seguimiento.

En un trabajo reciente en el que se pregunta a los clínicos sobre los pasos a seguir en un proceso de toma de decisiones compartida, se establecen tres posibles etapas. Una primera consistente en la exposición de las diferentes opciones, seguida de una fase de discusión de las mismas, para, por último, deliberar basándose en las preferencias de los pacientes (figura 1) (Elwyn, 2017). 


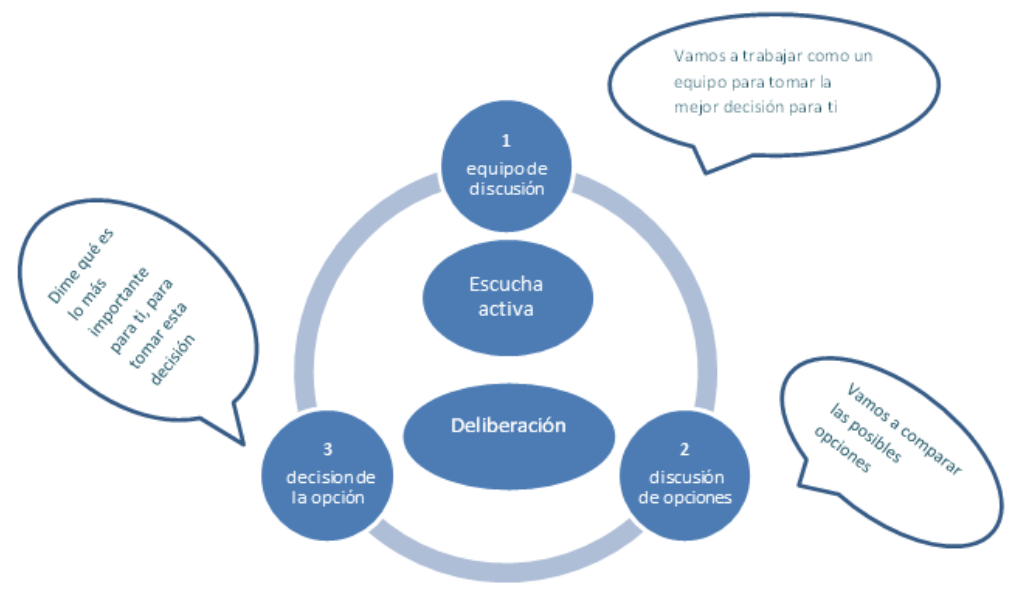

Figura 1. Etapas en el proceso de deliberación compartida. Adaptado de Elwyn (2017).

Para facilitar que el paciente se implique en un proceso de decisiones compartidas es necesario que esté informado. Hace décadas que se aborda el tema de la comunicación y la información al paciente atendiendo a su dificultad y a las consecuencias que se pueden derivar de una acción inadecuada (Butow, 1995; Baile, 2000). Se han descrito protocolos que facilitan el proceso informativo y la comunicación en medicina (Fallowfield, 1998) y se han promovido cursos estructurados para profesionales, con muy buenos resultados y cambios en la práctica clínica (Baile, 2000). El acto informativo debe respetar las diferencias individuales y las necesidades de información de cada paciente, en relación con estilos de afrontamiento ante la información amenazante y niveles de malestar emocional (Williams-Pichota, 2005; Timmermans, 2007). Cuando la información se adapta a las necesidades del paciente se consiguen mejores resultados de afrontamiento de la enfermedad y mayor adherencia al tratamiento (Joosten, 2008; Goss, 2013; Elwyn, 1999). Por el contrario, cuando se omiten las preferencias de los pacientes se observa que los tratamientos son menos efectivos y aumenta de forma injustificada la variabilidad clínica, que, en esta circunstancia, queda sujeta exclusivamente a criterio médico (Emanuel, 1992).

La decisión compartida busca ayudar a los pacientes a empoderarse en las decisiones que conciernen a su salud, objetivo último de la atención centrada en el paciente. El modelo de decisión compartida se propone como un método para conseguir aumentar la autonomía del paciente, su satisfacción, el control sobre la toma de decisiones y la adherencia con la decisión tomada al mismo tiempo que se reducen los costes en salud (Britten, 2000). 


\section{Las herramientas de ayuda a la toma de decisiones. Qué son y cómo se elaboran}

Para facilitar la implicación de la población en las decisiones que afectan a su salud, se han desarrollado las herramientas de ayuda para la toma de decisiones compartidas (HATD), conjunto de intervenciones complementarias utilizadas para informar y asesorar a los pacientes desde la evidencia científica (Ruiz Azarola, 2012; Perestelo-Pérez, 2013; Peralta, 2010; Ruiz Moral, 2012). Ante la creciente aparición de HATD, en 2003 se crea un grupo colaborativo que determina los criterios estándar para su elaboración, o criterios IPDAS que han sido actualizados desde su creación (del inglés International Patient Decisions Aid Standards) (Volk, 2013; O'Connor, 2005). Estos establecen los tres bloques que deben incluir las HATD: contenido clínico, proceso de desarrollo y evaluación de la eficacia (Elwyn, 2012).

Estas normas incluyen la exigencia de que la información contenida en las herramientas sea completa, esté actualizada, se base en fuentes de información de calidad probada y los autores declaren su financiación y conflicto de intereses (Elwyn, 2006).

Las HATD pueden presentarse mediante diferentes medios y formatos (folletos, texto escrito, vídeos, aplicaciones informáticas), utilizan diversos recursos como tablas en las que se presentan todas las opciones juntas y se explican las distintas características de cada una de ellas, y se sirven de ayudas visuales o pictogramas (Britten, 2000).

Existe suficiente evidencia científica de que las ayudas a la decisión, en comparación con la atención habitual, mejoran el conocimiento del paciente acerca de las opciones y reducen su conflicto a la hora de tomar una decisión, ya que se siente más informado y seguro del encaje de las opciones en sus valores personales (Abellán, 2008). Asimismo, existe evidencia moderada de que estas ayudas estimulan a las personas a tomar un papel más activo en la toma de decisiones, y de que incluir las probabilidades en las ayudas a la decisión contribuye a una percepción del riesgo más exacta (Mira, 2006). Pero, por otro lado, no hay evidencia científica de calidad en cuanto a que las ayudas a la toma de decisiones mejoren la congruencia entre la opción elegida y los valores del paciente (Charles, 2011). Igualmente, faltan estudios sobre los efectos de las HATD en la adhesión de los pacientes a la opción elegida, sobre el uso de estas herramientas en poblaciones con menor alfabetización y sobre el coste-efectividad de su uso (Charles, 2005). Después de la primera revisión Cochrane sobre las HATD de hace algunos años (Stacey, 2014), se dispone de una revisión Cochrane actual sobre el tema, aunque 
todavía muchas preguntas permanecen sin respuesta (Stacey, 2017). Se necesita evidencia adicional para estimar en qué medida estas herramientas mejoran la situación de los pacientes y en qué sentido lo hacen, si en el terreno intelectual, emocional y/o práctico (Elwyn, 2010). Tampoco está resuelta la cuestión de qué formatos serían más efectivos (SMS, vídeo, instrucciones orales, aplicaciones de software), qué técnicas resultarían más eficaces para cambiar pautas de comportamiento, y cómo influyen las características de la población en la efectividad de las intervenciones (Izquierdo, 2011). Asimismo, no se ha demostrado que las HATD reduzcan consistentemente el uso de tratamientos más invasivos o más costosos. Por lo tanto, la cuestión de si estas herramientas pueden reducir los costes en salud sigue sin estar clara. Lo que sí está claro es que las HATD no se deben usar para corregir problemas del sistema de salud como sobrecarga asistencial, inexperiencia de los clínicos y falta de recursos materiales y/o personales, entre otros (Montori, 2017).

En España, el desarrollo de estas herramientas y la promoción de la toma de decisiones compartida está siendo impulsada desde algunos servicios autonómicos de salud e incorporada en sus carteras de servicios con el fin de favorecer la capacitación y el fortalecimiento de los usuarios. En esta línea, durante los últimos años, la Agencia de Calidad del SNS (Plan de Calidad para el Sistema Nacional de Salud, 2010; Segura, 2010) ha financiado la elaboración y la validación de varias herramientas de ayuda a la toma de decisiones compartida por los servicios y agencias de evaluación de tecnologías sanitarias de Andalucía (AETSA), Canarias (SESCS), Cataluña (AQUAS) y Madrid (Hermosilla-Gago, 2008; Perestelo-Pérez, 2011; Perestelo-Pérez, 2010, Izquierdo, 2011).

Las situaciones ideales para el uso de las HATD en el ámbito de la salud son aquellas en las que existen dudas razonables sobre cuál es la mejor opción. Una de estas situaciones es la que concierne a la paciente sometida a una mastectomía por cáncer de mama cuando se plantea la conveniencia - o no- de una reconstrucción mamaria. En este caso, la reconstrucción es una alternativa terapéutica, aunque no es generalizable a todas las pacientes (Sirgo, 2011; De la Flor, 2015). En esta circunstancia, lo deseable es acompañar a la mujer en la toma de decisiones mediante un proceso compartido bien elaborado, que recabe su opinión y le proporcione información inteligible y personalizada para que, al final, sienta la decisión como propia y se perciba segura y satisfecha con la opción elegida.

La finalidad de este artículo es mostrar el proceso sistemático de desarrollo, resultado e impacto social de una HATD en el contexto de una mujer recién diagnosticada de cáncer de mama con indicación de mastectomía. 


\section{De la teoría a la práctica. Herramienta de ayuda a la toma de decisiones para mujeres con cáncer de mama e indicación de mastectomía}

Más de un 30\% de las mujeres que son diagnosticadas de un cáncer de mama deben someterse a una mastectomía (Pusic, 2012; Rusby, 2010). En estos casos, al impacto por el diagnóstico del cáncer se añade un tratamiento quirúrgico que en muchas ocasiones conlleva episodios de ansiedad relacionados con la apariencia, la sexualidad y la autoestima de las mujeres afectadas (Grogan, 2017). Ante esto, se pueden ofrecer diferentes opciones: reconstrucción inmediata, reconstrucción diferida o no reconstrucción de la mama. Con el objetivo de ayudar y empoderar a las mujeres en el proceso de elección de la opción terapéutica más acorde con sus preferencias, valores y expectativas, un equipo multidisciplinar de profesionales vinculados a la Universitat Rovira i Virgili de Tarragona creó una HATD digital para mujeres con cáncer de mama con indicación de mastectomía y posibilidad de reconstrucción.

A continuación se expone el proceso sistemático de desarrollo y resultado de esta HATD, así como el impacto social generado. Esta HATD ha sido diseñada y desarrollada teniendo en cuenta los criterios IPDAS y las etapas descritas por la Agencia de Evaluación, Tecnologías y Prestaciones del Sistema Nacional de Salud (SNS) para la evaluación y validación de una HATD (figura 2).

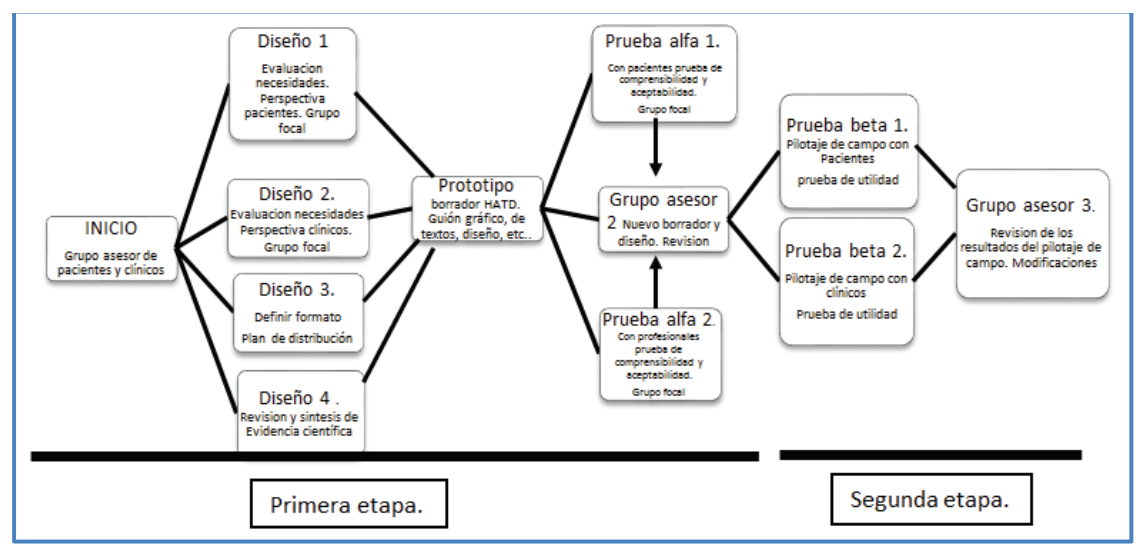

Figura 2. Modelo del Proceso de desarrollo de la HATD digital en mujeres con cáncer de mama, basado en el informe publicado por la Agencia de Evaluación, Tecnologías y Prestaciones del Sistema Nacional de Salud (SNS) para la evaluación y validación de una HATD. 


\section{Diseño 1 (Necesidades de los pacientes desde su perspectiva)}

En una primera etapa, una vez definido el alcance, propósito y público al que iría dirigida la HATD, se diseñó un estudio prospectivo de diseño cualitativo (diseño 1). Su objetivo fue explorar las experiencias, vivencias y necesidad de información y apoyo ante la toma de decisiones de un grupo de mujeres que, en el último año, habían sido diagnosticadas de cáncer de mama y tratadas con una mastectomía con reconstrucción inmediata, diferida o sin reconstrucción.

Las acciones de recogida de información se llevaron a cabo mediante entrevistas en profundidad (cuatro pacientes de rango de edad entre los 39 y los 68 años) y dos grupos focales (de 8 mujeres cada uno) con un guion preestablecido que incluía preguntas relacionadas con la experiencia ante la operación, la información recibida previa a la cirugía, la experiencia tras la intervención, cicatrices, efectos secundarios, complicaciones, secuelas o cumplimiento de expectativas, entre otras. Asimismo, se preguntó por el formato ideal de una HATD.

Del análisis surgieron seis categorías útiles para la construcción de la herramienta. Fueron las siguientes:

1. El impacto del diagnóstico del cáncer.

2. Información recibida ante la intervención.

3. Las experiencias en el posoperatorio.

4. Cumplimiento de expectativas.

5. Satisfacción con la decisión tomada.

6. Formato ideal de una HATD.

Las mujeres manifestaron un gran estrés emocional ante el diagnóstico, necesidades de información no cubiertas, posoperatorio más doloroso de lo esperado en algunos casos, expectativas poco realistas ante los resultados estéticos y dudas sobre la decisión tomada.

\section{Diseño 2 (Necesidades de los pacientes desde la perspectiva de los clínicos)}

A continuación, se invitó a un grupo de 8 clínicos a participar en un grupo focal. El grupo estuvo compuesto por una enfermera de planta de hospitalización, una auxiliar de enfermería de la consulta de cáncer de mama, un médico ginecólogo de la unidad de patología mamaria, un radioterapeuta, un oncólogo clínico, un médico de medicina nuclear especialista en ganglio centinela, una enfermera de hospital de día y una enfermera de la consulta de patología mamaria.

El objetivo fue conocer sus experiencias en relación con las necesidades de información y dificultades que presentaban las pacientes diagnosticadas de un cán- 
cer de mama. También se les preguntó cómo querrían que fuera la HATD. Las categorías que surgieron tras el análisis de las declaraciones de este grupo fueron:

1. Necesidad de trabajo en equipo centrado en el cuidado a la mujer y sus necesidades.

2. Modelos de atención: medicina paternalista versus decisiones compartidas

3. Dificultades a la hora de informar a estas mujeres.

4. Formato ideal de una HATD.

Los profesionales manifestaron que, ante esta situación, la mujer necesita recibir una información real, ecuánime y adaptada para poder tomar la decisión que mejor responda a su situación clínica, valores, necesidades y preferencias, para así afrontar de un modo más adecuado su recuperación. Mostraron su convencimiento de que una HATD creada para tal fin permitiría la participación de la mujer en la decisión, mejoraría la satisfacción y disminuiría el conflicto decisional.

\section{Diseño 3 (Formato y plan de distribución)}

Tras la transcripción y análisis de los discursos de las entrevistas y grupos focales, se concretaron los aspectos más importantes a considerar en la construcción de la HATD, en relación con el formato, el nivel de contenidos, la presencia de imágenes explicativas, así como la distribución de la misma.

Ante el diagnóstico del cáncer las mujeres presentaron emociones diversas. Recibirlo les produjo llanto, miedo y emociones que quedan reflejadas en sus relatos. Algunas inicialmente respondieron con incredulidad y negación. Sobre la información recibida ante la intervención antes de la mastectomía, la mayoría de las mujeres explicó que confiar en la opinión y consejo del equipo médico se presentó como una prioridad, la información recibida se consideró buena y suficiente. Las mujeres manifestaron estar satisfechas. Algunas mujeres necesitaron ampliar la información. Pero comprobamos que es posteriormente, una vez superada la convalecencia de la intervención, cuando aparecen más quejas. En cuanto a las experiencias en el posoperatorio, las vivencias recogidas fueron muy diversas. Estuvieron mayoritariamente relacionadas con las complicaciones posteriores y fue sobre todo el dolor el que estuvo presente en la mayoría de los relatos. Las expectativas sobre la reconstrucción inmediata estuvieron muy relacionadas con los resultados estéticos finales. Así, encontramos tres subcategorías que son: los símbolos sociales del pecho, la estética final y las cicatrices. Por último, en cuanto a la satisfacción con la decisión tomada aparecen dos tipos de posicionamientos, las que sí volverían a pasar por lo mismo a pesar de las complicaciones porque lo 
valoran como algo positivo que se aproxima a satisfacer sus necesidades y las que dan mucho valor a los inconvenientes y no se lo volverían a hacer.

\section{Diseño 4 (Revisión y sintesis de la evidencia científica)}

En una segunda etapa, se realizó una nueva revisión bibliográfica en la que se buscaron artículos relacionados con el cáncer de mama y las HATD en PubMed, CINAHL, PsychLIT, PsycINFO, IBECS y Cochrane library plus, de octubre 2014 a abril 2015. Los descriptors utilizados fueron: patient decision aid; patient decision making; shared decision making; breast cancer and breast reconstruction; mastectomy. La búsqueda se centró en la utilización de HATD en cáncer de mama, cómo confeccionarlas, formatos y sistema de evaluación de las mismas. Se incluyeron artículos de revisión y originales en español e inglés, publicados entre enero de 2000 y abril de 2015.

\section{Prototipo de la HATD}

Finalmente se optó por una HATD digital para Internet, lineal, dinámica y totalmente interactiva, que ofrecía apoyo deliberativo activo, información veraz y contrastada, dibujos de las diferentes opciones terapéuticas y un simulador de imágenes en función de la tipología de las mamas de cada paciente.

\section{Pruebas Alfa 1 y 2}

El prototipo de la herramienta fue revisado por pacientes que habían pasado por experiencias similares, que posteriormente fueron entrevistadas. Se presentó también al Consejo Consultivo de Pacientes de Cataluña. Las sugerencias y cambios propuestos fueron posteriormente revisados y valorados y en la mayoría de los casos incorporados en la herramienta.

\section{Pilotaje de Campo Beta 1 y 2}

Actualmente, la HATD elaborada está siendo probada en el marco de un estudio observacional que contempla la opinión de pacientes y médicos ajenos al desarrollo de la HATD.

\section{La berramienta}

La HATD fue finalmente publicada en junio de 2015 como página web de acceso libre para profesionales y pacientes en catalán y castellano. Desde el mes de noviembre de 2016, y gracias a un convenio entre el Centro de Transferencia de Tecnología e Innovación (CTTi) de la Fundación URV, el Instituto de Investi- 
gaciones Sanitarias Pere Virgili y la Agencia de Calidad y Evaluación Sanitarias (AQUAS) de la Generalitat de Cataluña, la aplicación está disponible en la web Decisiones Compartidas del Departament de Salut de la Generalitat en este enlace: <http://decisionscompartides.gencat.cat/ca/decidir-sobre/reconstrucciomamaria-despres-de-cirurgia-per-cancer $/>$.

La HATD se estructuró en tres bloques. En el primero, «El estudio. Te queremos ayudar», se explica brevemente qué es una HATD. A través del segundo apartado se accede al núcleo central de la HATD. Por último, en el tercer apartado, «Más información», aparecen preguntas sobre el cáncer de mama contestadas por profesionales, enlaces a organizaciones relacionadas con el tema y una dirección de correo electrónico para poder contactar (figura 3).

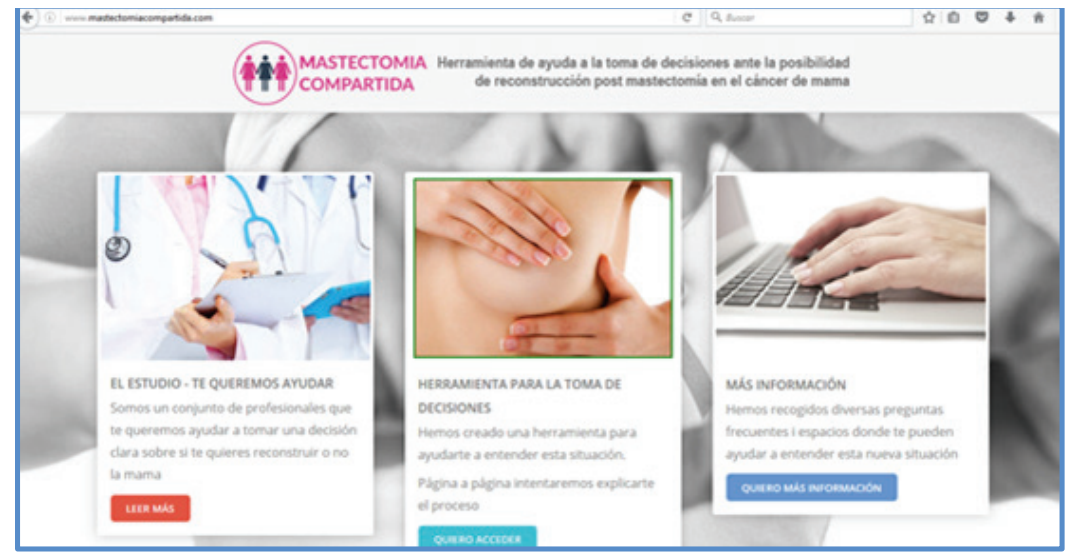

Figura 3. Pantalla presentación HATD.

En el segundo bloque, la HATD propiamente dicha, se explican, a través de tablas comparativas, las tres posibilidades que existen ante una mastectomía: no reconstruir, reconstrucción inmediata o reconstrucción diferida. Se presentan las diferentes técnicas reconstructivas y los resultados estéticos, y se informa sobre los días de ingreso en cada situación, la duración de las intervenciones, aspectos del posoperatorio y las posibles complicaciones (figura 4). 


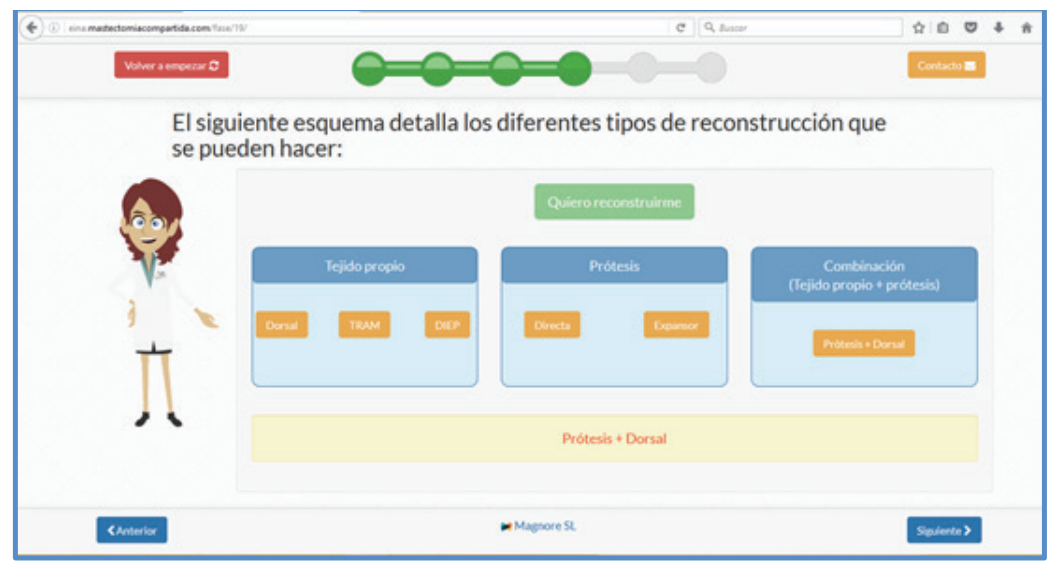

Figura 4. Diferentes tipos de reconstrucción.

Las pantallas siguientes de este bloque tratan de crear expectativas realistas sobre la simetría, el tamaño y la sensibilidad de las mamas ante una reconstrucción. También se tiene la opción de acceder a testimonios de pacientes en las distintas situaciones. Finalmente, se accede a un simulador de imágenes con tres posibles fenotipos de torsos femeninos y se invita a la mujer a identificarse con uno de ellos con el objetivo de conseguir que se represente, de la forma más fidedigna posible, el resultado final en su caso (figura 5).

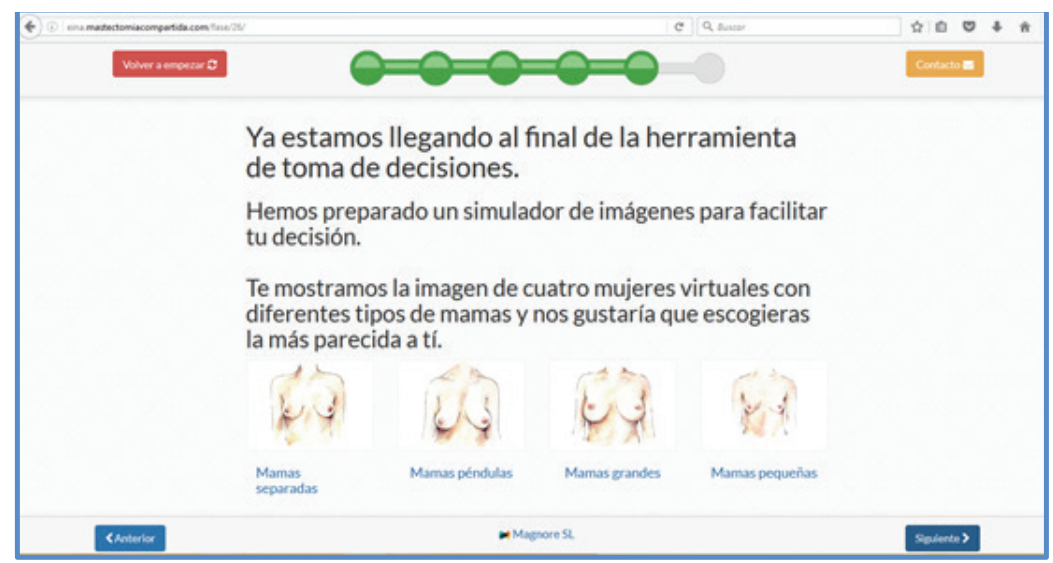

Figura 5. Simulador de imágenes. 


\section{Impacto del resultado}

La oportunidad de este proyecto es su potencial impacto social. La HATD mostrada fue galardonada con el Premi Consell Social URV al Impacto Social de la Investigación, en el ámbito de las Ciencias de la Salud y de la Vida, en su modalidad ex post, en su convocatoria de 2017, <http://wwwa.urv.cat/ogovern/ consellsocial/PISR/Acta2017.pdf $>$.

Esta herramienta informática con simulador podrá ser utilizada por cualquier mujer afectada por un cáncer de mama con indicación de mastectomía que tenga acceso a Internet y desde cualquier sitio. También podrá ser utilizada para reforzar la información que dan los profesionales en la consulta de centros sanitarios (unidades de patología mamaria de centros hospitalarios públicos o privados en España o países con usuarios con características culturales y sistemas sanitarios similares - Europa y América Latina-), Administración pública sanitaria (Departamento de Salud de la Generalitat de Cataluña, Sistema Nacional de Salud, etc.), seguros médicos privados con unidades de patología mamaria y/o universidades que quieran incluir en su currículum este conocimiento.

Actualmente se está llevando a cabo un estudio para determinar el impacto, en términos de satisfacción con la decisión tomada, que está teniendo la herramienta en nuestras pacientes, comparando diversos ítems entre el grupo que la utiliza y el grupo a la cual no se le ha mostrado.

Además, el impacto socioeconómico de la utilización de esta HATD podría repercutir en nuestro sistema sanitario. En primer lugar, al elevar la calidad de vida del paciente y favorecer el empoderamiento del ciudadano. En segundo lugar, y a pesar de que no hay evidencia sobre ello, se ha sugerido que las HATD podrían favorecer la optimización de costes en los procedimientos y recursos sanitarios que elige el usuario final, puesto que se ha demostrado que cuando el paciente es requerido como participante en la toma de decisiones sobre su salud, suele inclinarse por las opciones más sencillas, tanto en términos de procedimiento como de costes (Montori, 2017). Por último, la previsible disminución de demandas del paciente contra el sistema sanitario aparece como una nueva derivada de la utilización de las HATD en la atención a la salud.

La construcción de las HATD es, en realidad, un acto de cocreación, porque parte de las necesidades y preocupaciones que formula el usuario mediante técnicas y procedimientos de investigación para los que es requerido, sus inquietudes y sugerencias son incorporados en el diseño de las herramientas, y vuelven a él cuando se le pide conformidad en la fase de validación.

Esta herramienta ha servido de base para la creación de nuevas herramientas en otros campos de salud sobre los que se está trabajando y los diversos Gobier- 
nos de las distintas comunidades autónomas ya disponen en sus páginas web de apartados para las «decisiones compartidas» que incluyen estos instrumentos. El futuro de las relaciones médico-paciente pasará inexorablemente por centrar las necesidades individuales de cada paciente como el núcleo de las decisiones diagnóstico-terapéuticas. Por este motivo, pensamos que es imprescindible disponer de herramientas que faciliten el proceso, y nuestra herramienta es un ejemplo de esto.

\section{Bibliografía}

Abellán, J. M.; Sánchez Martínez, J. E. Y Martínez Pérez (2008). "¿Cómo Incorporar las preferencias de los pacientes en la toma de decisiones clínicas?». Gac Sanit, 22(supl. 1), 179-85.

Baile, W. F; Buckman, R.; Lenzi, R.; Glober, G.; Beale, E. A. Y Kudelka, A. P. (2000). «SPIKES-A six-steps protocol for delivering bad news: Application to the patient with cancer». Oncologist, 5, 302-311.

Britten, N.; Stevenson, F. A.; Barry, C.; Barber, N. y Bradley, C. P. (2000). «Misunderstandings in prescribing decisions in general practice: qualitative study». BMJ, 320, 484-488.

Butow, P. N.; Dunn, S. M. y Tattersall, M. H. (1995). «Communication with cancer patients: does it matter?» J Palliat Care, 11, 34-38.

Charles, C. y Gafni, A. (2011). "Shared treatment decision-making and the use of decision-aids». En: Kissane, D. W.; Bultz, B. D.; Butow, P. H. y Finlay, I. G. Handbook of Communication in Oncology and Palliative Care (pp. 41-50). New York: Oxford University Press.

Charles, C.; Gafni, A. y Whelan T. (1997). "Shared decision-making in the medical encounter: what does it mean? (Or it takes at least two to tango)». Soc Sci Med, 44, 681-692.

Charles, C.; Gafni, A.; Whelan, T. y O’Brien, M. A. (2005). «Treatment decision aids: conceptual issues and future directions». Health Expect, 8(2), $114-125$.

Coucerro, A. (2005). «Dinámica de la decisión clínica. ¿Hay métodos que ayuden a incorporar los valores del paciente en la toma de decisiones clínicas?» JANO 21, 27(1.581), 86-88.

De la Flor López, M.; De Molina Fernández, I.; Sirgo Rodríguez, A. y Montes Muñoz, M. J. (2015). «Reconstrucción inmediata en cáncer de mama+¿Las mujeres están satisfechas con su decisión?»Psicooncología, 12(2/3), $393-403$. 
Elwyn, G.; Durand, M. A.; Song, J.; Aarts, J; Barr, P. J.; Berger, Z.; HaN, P. K. et alii (2017). «A three-talk model for shared decision making* multistage consultation process». Bmj, 359, j4891.

Elwyn, G.; Edwards, A. y Kinnersley, P. (1999). «Shared decision-making in primary care: the neglected second half of the consultation». $\mathrm{Br} J \mathrm{Gen}$ Pract, 49, 477-482.

Elwyn, G.; Frosch, D.; Thomson, R.; Joseph-Williams, N.; Lloyd, A.; Kinnersley, P; Edwards, A. et alii (2012). "Shared decision making* a model for clinical practice».J Gen Intern Med, 27(10), 1.361-1.367.

Elwyn, G.; Frosch, D.; Volandes, A. E.; Edwards, A. y Montori, V. M. (2010). "Investing in deliberation: a definition and classification of decision support interventions for people facing difficult health decisions ». Med Decis Making, 30(6), 701-711.

Elwyn, G.; O’connor, A.; Stacey, D.; Volk, R.; Edwards, A.; Coulter, A.; Butow, P. et alii (2006). «Developing a quality criteria framework for patient decision aids: online international Delphi consensus process». Bmj, 333(7.565), 417.

Elwyn, G.; Edwards, A.; Kinnersley, P. y Grol, R. (2000). «Shared decision making and the concept of equipoise: the competences of involving patients in healthcare choices». Br J Gen Pract, 50, 892-99.

Emanuel, E. J. y Emanuel, L. L. (1992). «Four models of the physician-patient relationship».J Am Med Assoc, 267, 2.221-2.226.

Fallowfield, L.; Lipkin, M. y Hall, A. (1998). "Teaching senior oncologist communication skills: results from phase I of a comprehensive longitudinal program in the United Kingdom». J Clin Oncol, 16, 1.961-1.968.

Goss, C.; Ghilardi, A.; Deledda, G.; Buizza, C.; Bottacini, A.; Del Piccolo, L; Bighelli, I. et alii (2013). «Involvement of breast Cancer patients during oncological consultations: a multicentre randomised controlled trial-the INCA study protocol». BMJ open, 3(5), e002266.

Grogan, S. y Mechan, J. (2017). «Body image after mastectomyः A thematic analysis of younger women's written accounts».J Health Psychol, 22(11), $1.480-1.490$.

Guyat, G. H. (1991). «Evidence-Based Medicine» [editorial]. ACP J Club A-16, 114(supl. 2), A16.

Hermosilla-Gago, T*; Vidal-Serrano, S*y Buzón-Barrera, M. L* (2008). Herramienta de ayuda a la toma de decisiones (HATD) para pacientes con fibrilación auricular no valvular. Sevilla: AETSA. 
Izquierdo, F*; Gracia, J.; Guerra, M.; Blasco, J. A. y Andradas, E. (2011). «Health technology assessment-based development of a Spanish breast cancer patient decision aid». Int J Technol Assess Health Care, 27(4), 363-368.

Joosten, E. A.; De Fuentes-Merillas, L.; De Weert, G. H.; Sensky, T; Van Der Staak, C. P. F. y De Jong, C. A. (2008). «Systematic review of the effects of shared decision-making on patient satisfaction, treatment adherence and health status». Psychother Psychosoms, 77(4), 219-226.

May, V. M.; Montori, V.y Mair, F. S. (2009). «We need minimally disruptive medicine». BMJ, 339, b2803.

Mendoza, P. S.; Jofré, A. V. y Valenzuela, S. S. (2006). «La toma de decisiones en salud y el modelo conceptual de Ottawa». Invest Educ Enfer, 24(1), $86-92$.

Mira, J. J*; Lorenzo, S.; Pérez-Jover, V. y Rodríguez-Marín, J+ (2006). «No estar satisfecho no equivale a estar insatisfecho». Rev Clin Esp, 206, 209-210.

Mitchell, P. H. (2008). Defining Patient Safety and Quality Care». En: Hughes, R. G. (ed.).Patient Safety and Quality: An Evidence-Based Handbook for Nurses. Rockville (MD). US: Agency for Healthcare Research and Quality. Montori, V. M.; Kunneman, M. y Brito, J. P. (2017). «Shared Decision Making and Improving Health Care: The Answer Is Not In».JAMA, 318(7), 617-618.

O'Connor, A. M. y Jacobsen, M. J. (2003). Workbook on developing and evaluating patient decision aids. Ottawa: Health Research Institute. Disponible en: <www.ohri.ca/decisionaid >.

O’Connor, A. M.; Jacobsen, M. J. y Stacey D. (2002). «An evidence-based approach to managing women's decisional conflict».J Obstet Gynecol Neonatal Nurs, 31(5), 570-581.

O’Connor, A.; Llewellyn-Thomas, H. y Stacey, D. (2005). International Patient Decision Aid Standards (IPDAS) Collaboration IPDAS Collaboration Background Document. Disponible en: <http //ipdas.ohri.ca/IPDAS_ Background.pdf $>$.

Peralta Munguía, L. (2010). La participación del paciente en la toma de decisiones en las consultas de atención primaria (tesis doctoral). Santander: Universidad de Cantabria. Disponible en: <http://www.tdx.cat/bitstream/handle/10803/10705/TesisLPM.pdf>.

Perestelo-Pérez, L.; Pérez-Ramos, J.; González-Lorenzo, M.; RiveroSantana, A. y Serrano-Aguilar, P. (2010). «Decision aids for patients facing health treatment decisions in Spain: preliminary results». Patient Educ Couns, 80(3), 364-371. 
Perestelo-Pérez, L.; Pérez-Ramos, J; Rivero-Santana, A.; Carballo-González, D. y Serrano-Aguilar, P. (2013). Manual con criterios de evaluación y validación de las Herramientas de Ayuda para la Toma de Decisiones. Disponible en: http //ipdas.ohri.ca/SESCS_HATD.pdf.

Perestelo-Pérez, L; Rivero-Santana, A.; Pérez-Ramos, J; GonzálezLorenzo, M.; Gracia-San Román, J. y Serrano-Aguilar, P. (2011). «Shared decision making in Spain: current state and future perspectives». $Z$ Evid Fortbil Qual Desundbwes, 105(4), 289-295.

Plan de Calidad para el Sistema Nacional de Salud. Ministerio de Sanidad, Politica Social e Igualdad. Publicación Web (2010). Disponible en: <http://www.mspsi.es/organizacion/sns/planCalidadSNS/pdf/pncalidad/PlanCalidad2010. pdf $>$.

Pusic, A. L.; Klassen, A. F.; Snell, L.; Cano, S. J*; McCarthy, C.; Scott, A.; Cordeiro, P. G. et alii (2012). «Measuring and managing patient expectations for breast reconstruction: impact on quality of life and patient satisfaction». Expert Rev Pharmacoecon Outcomes Research, 12(2), 149-158.

Ratliff, A.; Angell, M;; Dow, R.W; Kuppermann, M.; Nease, R. F; Fisher, R.; Sox, H. C. et al. (1999). «What is a good decision». Eff Clin Pract, 2(4), 185-197.

Ruiz-Azarola, A. y Perestelo-Pérez, L+ (2012). «Participación ciudadana en salud: formación y toma de decisiones compartida. Informe SESPAS». Gac Sanit, 26, 158-161.

Ruiz Moral., R.; Peralta Munguía, L.; Pérula de Torres, L; Olloqui Mundet, J.; Carrión de la Fuente, T.; Sobrino López, A.; MartíNez LeChugA, M. et alii (2012). «Opiniones y percepciones de los pacientes sobre su participación en la toma de decisiones en las consultas de medicina de familia». Atención Primaria, 44(1), 5-12.

Rusby, J. E.; Waters, R. A.; Nightingale, P. G. y England, D. W. (2010). «Immediate breast reconstruction after mastectomyः what are the long-term prospects?» Annals Rl Coll Surg England, 92(3), 193-197.

Segura, A. (2010). «La participación ciudadana, la sanidad y la salud. Gestión y Clinica Sanitaria, 12(2), 55-62.

Sirgo, A, (2011). «Toma de decisiones compartidas en Oncología: Reconstrucción inmediata tras una mastectomía» (Capítulo 11). En: Gil Moncayo, F. Counselling y Psicoterapia en Cáncer. Barcelona: Elsevier.

Stacey, D;; Légaré, F.; Col, N. F*; Bennett, C. L*; Barry, M. J*; Eden, K. B.; Wu, J. et alii (2014). «Decision aids for people facing health treatment or screening decisions». Cochrane Database of Syst Rev, Issue 1. Art. No.: CD001431. DOI: 10.1002/14651858.CD001431.pub4. 
Stacey, D; Légaré, F. y Lewis, K. B. (2017). «Patient decision aids to engage adults in treatment or screening decisions»+JAMA, 318(7), 657-658.

Stiggelbout, A. M.; Van der Weijden, T; De Wit, M. P.; Frosch, D; Légaré, F.; Montori, V. M.; Elwyn, G. et alii (2012). «Shared decision making: really putting patients at the centre of healthcare». BMJ, 344, e256.

Timmermans, L. M*; Van Zuuren, F. J*; Van der Maazen, R. W. M.; Leer J. W. H. y KraAimaat, F. W. (2007). «Monitoring and blunting in palliative and curative radiotherapy consultations». Psychooncology, 16(12), 1.1111.120 .

Volk, R. J*; Llewellyn-Thomas, H.; Stacey, D. y Elwyn, G. (2013). «Ten years of the International Patient Decision Aid Standards Collaboration: evolution of the core dimensions for assessing the quality of patient decision aids». BMC Med Inform Decis Mak, 13(supl. 2), S1-6947-13-S2-S1.

Williams-Pichota, P; Pizarro, J*; Schneider, T. R.; Mowad, L. y Salovey, P. (2005). «Matching health messages to monitor-blunter coping styles to motivate screening mammography». Health Psychol, 24(1), 58-67. 